\title{
Proceso de paz y desafíos regionales en Colombia
}

\section{Peace Process and Regional Challenges in Colombia}

\author{
Fernando Harto de Vera ${ }^{1}$ \\ Universidad Complutense de Madrid (España)
}

Recibido: 11-11-17

Aprobado: 26-01-18

\section{Resumen}

El objetivo del presente artículo es tratar la territorialización del proceso de paz colombiano. Se trata de poner de manifiesto la ineludible necesidad de que para la implementación de los Acuerdos de Paz es necesario tener en cuenta el escenario en el que se van a llevar a cabo las acciones, actuaciones y políticas públicas de paz. Colombia es una realidad conformada por una rica diversidad de realidades socioespaciales y por ello cualquier estrategia de paz que no tenga en cuenta esta pluralidad está condenada al fracaso.

El análisis que se presenta en estas páginas está organizado en torno a tres ejes. En primer lugar, desde una perspectiva epistemológica, se aborda la relevancia del territorio como categoría de análisis. En segundo lugar, se describe cuál es el escenario del que estamos tratando, esto es, la configuración del territorio colombiano y su ordenamiento. Una vez establecidas las coordenadas en las que nos desenvolvemos se procederá a una lectura analítica de la agenda de paz en clave territorial, es decir, poner de manifiesto los retos y problemáticas que la aplicación de los acuerdos de paz va a suponer en función de las características territoriales del país. Finalmente, se cierra la reflexión con un apartado de recapitulación y conclusiones.

Palabras-clave: Paz en Colombia, Territorialización, Acuerdos de La Habana.

\footnotetext{
${ }^{1}$ (fernandoharto@cps.ucm.es). Doctor en Ciencias Políticas y Sociología de la Universidad Complutense de Madrid y Profesor Titular de la Facultad de Ciencias Políticas y Sociología de la Universidad Complutense de Madrid, España.
} 


\begin{abstract}
The objective of this article is to deal with the territorialization of the Colombian peace process. It is to highlight the unavoidable need of taking into account the scenario where are the public policies will take place. Colombia is a reality shaped by a rich diversity of socio-spatial realities and therefore any peace strategy that does not take into consideration allow for this plurality is doomed to failure.

The analysis presented in these pages is organized around three axes. First, from an epistemological perspective, the relevance of the territory as a category of analysis is addressed. Secondly, it describes what the scenario we are dealing with is, which is the colombian territory configuration. Once the coordinates in which we live are established, an analytical reading of the peace agenda will be carried out in a territorial key which means to highlight the challenges and problems that the implementation of the peace agreements will entail in terms of the territorial characteristics of the country. Finally, the reflexion is closed with recapitulation and conclusions.
\end{abstract}

Key-words: Colombian Peace Process, Territorialization, Agreements of Havana.

\title{
1. Introducción
}

El objetivo del presente artículo es tratar la territorialización del proceso de paz colombiano. Se trata de poner de manifiesto la ineludible necesidad de que para la implementación de los Acuerdos de Paz es necesario tener en cuenta el escenario en el que se van a llevar a cabo las acciones, actuaciones y políticas públicas de paz. Colombia es una realidad conformada por una rica diversidad de realidades socioespaciales y por ello cualquier estrategia de paz que no tenga en cuenta esta pluralidad está condenada al fracaso.

El análisis que se presenta en estas páginas está organizado en torno a tres ejes. En primer lugar, desde una perspectiva epistemológica, se aborda la relevancia del territorio como categoría de análisis. En segundo lugar, se describe cual es el escenario del que estamos tratando, esto es, la configuración del territorio colombiano y su ordenamiento. Una vez establecidas las coordenadas en las que nos desenvolvemos se procederá a una lectura analítica de la agenda de paz en clave territorial, es decir, poner de manifiesto los retos y problemáticas que la aplicación de los acuerdos de paz va a suponer en función de las características territoriales del país. Finalmente, se cierra la reflexión con un apartado de recapitulación y conclusiones. 


\title{
2. La importancia del territorio: el giro espacial
}

Desde la crisis del behaviorismo, a partir de mediados de los años sesenta del siglo pasado, las Ciencias Sociales han experimentado varios giros epistémicos. El primero de todos ellos fue el giro lingüístico seguido a continuación del giro estético, el giro icónico y, por último, el más reciente de todos ellos, el giro espacial (Colom y Rivero 2015: 7).

La expresión "giro lingüístico" fue introducida por Gustav Bergmann en 1953 para referirse a la manera de hacer filosofía de Wittgenstein en su "Tractatus logico-philosophicus" caracterizada por un cambio metodológico sustancial que afirmaba la imposibilidad de filosofar sin hacer un análisis previo del lenguaje (Bergmann 1953). Posteriormente la expresión fue popularizada por Rorty en 1967 en su obra "El giro lingüístico" en el que la expresión significaba un giro hacia la filosofía del lenguaje (Rorty 1990). El concepto "giro epistémico" hizo fortuna y a partir de entonces traspasó las fronteras de la Filosofía extendiéndose a las ciencias sociales y fue empleado cada vez que se producía una nueva visión, una nueva aportación que por un lado cuestionaba las certezas, hábitos y formas de construir conocimiento tradicionales y al mismo tiempo abría nuevas perspectivas y horizontes.

El giro lingüístico llevó a una hegemonía del Texto como objeto de estudio y análisis. Sin embargo:

\begin{abstract}
"la hegemonía del Texto condujo a una serie de agotamientos de sus propias bases, que a su vez provocó una serie de resurrecciones de las bases que había ayudado a desestabilizar: de la obra, del cuerpo y del lugar. Una vez que se dio por agotada a la crítica que operaba únicamente en, desde y a través del Texto, apareció la necesidad urgente de dar forma al Texto mediante la obra, de identificar los sujetos que lo enuncian en un cuerpo, y finalmente de situar al Texto en un lugar determinado. En este ámbito puede ubicarse el giro espacial que experimentó la geografía, y en general las ciencias sociales, en el transcurso de los años ochenta a los noventa del siglo XX, en el de dar forma, identidad y ubicación a las prácticas sociales" (Quesada 2016: 155).
\end{abstract}

El giro espacial consiste en poner de manifiesto la capital importancia del espacio y el territorio como categorías conceptuales desde las que construir el conocimiento que las ciencias sociales nos aportan. Este movimiento data de finales de los años ochenta. Sin embargo, el proceso hasta llegar a ese momento se inicia con unos antecedentes que podemos cifrar en la década de los años 20 del pasado siglo con la Escuela de Chicago y su introducción de la categoría espacio en los Estudios Urbanos. Tras estos antecedentes habría que esperar cuarenta años y trasladarnos de escenario para encontrar el siguiente hito en este proceso de "espacialización": en el París sesentayochista emergen 
las figuras de Henri Lefebvre y Michel Foucault, padres fundadores del giro espacial (Soja 2009: 18).

Ambos autores partían de la afirmación de que la organización del espacio es una variable central para poder explicar coherentemente la estructura y funcionamiento del sistema capitalista (Warf and Arias 2009: 3). Pero, además, Lefebvre aporta una resignificación del concepto de espacio. Hasta ese momento, el espacio se entendía como algo neutral, objetivo y dado. Lefebvre rechaza esta visión cosificada y estable del espacio en nombre de una concepción del mismo como algo creado y recreado continuamente. El espacio es una categoría conceptual socialmente construida y dinámica que se ve afectada por la acción que se desarrolla en su interior. Así, en términos políticos el espacio dejó de ser considerado como un receptáculo de la praxis política para ser concebido como un objeto susceptible de intervención política (Velázquez 2013: 179). Es decir, en el giro espacial destacan dos elementos: a) la centralidad del espacio como categoría analítica y b) la resignificación de lo que se entiende por "espacio".

Por su parte, Foucault en su conocida conferencia "Los espacios otros"2plantea la paridad ontológica entre espacio y tiempo de tal modo que ambas dimensiones se conforman la una a la otra. Se pregunta porqué el tiempo se piensa en términos de "movimiento", "dinámica", "dialéctica", "desarrollo" o "proceso" mientras que el espacio es considerado como algo "fijo", "muerto", como un "telón de fondo", como el "contenedor" de los procesos sociales y la historia. Desde mediados del siglo XIX se ha generado una visión "historicista" en las ciencias sociales consistente en señalar que la voluntad humana se despliega en el tiempo y la historia, relegando al espacio y la geografía a la posición de un mero escenario en el que se produce el drama. Este "historicismo social" ha permeado todo el pensamiento occidental incluyendo al marxismo. Frente a esta situación, Foucault plantea que es necesaria una revalorización del espacio frente al tiempo a través del surgimiento de nuevas formas de pensar el espacio. Esta nueva manera de concebir el espacio será denominada por Foucault como "heterotopología" (Soja 2009: 18).

A través de la ruta que los padres fundadores del giro espacial, Lefebvre y Focault habían abierto, otros autores como David Harvey transitaron a partir de los años 70. Continuando con la línea marxista presente en los análisis de Lefebvre, el objetivo de Harvey consistió en hacer una lectura del marxismo desde la perspectiva del giro espacial logrando una espacialización del marxismo y una marxificación del espacio. Su análisis se centró en la estructura profunda de la producción de mercancías y en la realización de la mercancía en

\footnotetext{
${ }^{2}$ «Des espaces autres», conferencia pronunciada en el Centre d'Études architecturales de Túnez el 14 de marzo de 1967 y cuya publicación no fue autorizada por M. Foucault hasta 1984 en Architecture, Mouvement, Continuité, $\mathrm{n}^{\circ}$ 5, octubre 1984, págs. 46-49. Paris.
} 
dinero generando un modelo de producción y de proceso de trabajo que arrojó luz sobre su transformación en el tiempo y el espacio. Desde esta perspectiva, el paisaje se constituía en un marco que expresaba y reflejaba la lógica de la producción de la mercancía en un momento histórico determinado (Warf and Arias 2009: 3).

Tras la "fase marxista" del giro espacial la producción dentro de esta corriente recibió el impulso posmoderno de Edward Soja ya en la década de los noventa. Soja planteó que lo espacial no puede ser subordinado al tiempo o a lo social. Así sostiene que la teoría social debería descansar en la interacción entre tiempo, espacio y estructura social. Cada uno de estos tres elementos estructura y a la vez es estructurado por los otros. Asimismo, argumenta que el giro espacial ha significado tanto el fin del historicismo que habría privilegiado el tiempo sobre el espacio, como la reafirmación del espacio dentro de la teoría social. Las raíces de esta revalorización se encuentran en Kant que ya planteó que las dos dimensiones (tiempo y espacio) debían tener la misma importancia (Warf and Arias 2009: 3). En palabras de Boyer (2007: 160): "se trata más bien de buscar otras formas de articulación entre lo histórico y lo espacial, una en la que lo espacial no sea deducido de lo temporal, sino que tenga su propio régimen y autonomía. Es necesario superar "la época de la imagen histórica (cursivas de la autora) del mundo y desuturar la identidad entre historia y tiempo humano. El espacio no puede seguir subordinado al tiempo".

Este planteamiento de Soja se complementa con una llamada a la comunicación entre las distintas ciencias sociales basada en la espacialización de las mismas. Así encontramos que formula categorías conceptuales tales como "justicia espacial" o "democracia regional" como expresión de esa hibridación entre diferentes disciplinas, en este caso la geografía ("espacial", "regional") y la ciencia política ("justicia", "democracia"):

"En la década de 1990, se ha desarrollado un nuevo discurso crítico sobre las ciudades y las regiones en un intento de dar sentido práctico y teórico a las crisis emergentes de la postmetrópolis. Informado en términos teóricos por las epistemologías estructuralistas, el feminismo posmoderno, la teoría queer, los análisis postmarxistas, las críticas postcoloniales y otras críticas antirracistas [...] este discurso todavía en desarrollo ha desencadenado nuevos modos de pensar las especificidades particulares y espaciales de Los Ángeles y otras ciudades-región globalizadas. [...] el fomento de la democracia regional y la reducción de lo que he llamado metropolaridades gira principalmente en torno a la base impositiva regional o el reparto de la renta, el incremento del control sobre las políticas de inversión y la construcción de nuevas coaliciones políticas entre las viejas zonas residenciales y las ciudades centrales [...] Mientras que dichos llamamientos a la justicia redistributiva no son nuevos, lo que hoy es distinto es su relación explícita con la agenda regional basada en la comunidad y con una creciente conciencia pública de cómo el desarrollo geográficamente desigual 
y las especificidades espaciales de la economía urbana-regional reestructurada trabajan para generar y mantener las injusticias y las desigualdades económicas y sociales" (Soja 2008: 559, 560, 561).

\section{La configuración del territorio colombiano}

Como bien plantea Pécaut (2004: 25) "tanto por razones geográficas como por razones históricas Colombia nunca ha sido un territorio unificado sobre el cual el Estado haya ejercido una soberanía indiscutible". Desde los mismos inicios de la fundación del país, el propio Simón Bolívar se refería al proceso de construcción nacional en los siguientes términos: "La nación debe crearse desde arriba para evitar el caos" (Bolívar 2001: 309-318)³. Esta temprana visión se confirmará en la obra de historiadores contemporáneos como David Bushnell $(1996)^{4}$ quien se refiere a Colombia denominándola "una nación a pesar de si misma" o como Fernán González (2006) que la considera "una nación en construcción".

En este sentido, Colombia no difiere de la tendencia general del resto de América Latina que al abordar el proceso de construcción del Estadonación enfrentó serias dificultades que llevó a que en la práctica totalidad de los territorios independizados de la corona española dicho proceso tuviera un carácter inconcluso y exhibiera importantes carencias y debilidades. El liberalismo se convirtió en la base de los programas y teorías para la construcción de las antiguas colonias como estados independientes. Pero la experiencia del liberalismo en América Latina se caracteriza por el hecho de que las ideas liberales fueron aplicadas en naciones con un alto grado de estratificación social y racial y económicamente subdesarrolladas en las que se contaba con una amplia herencia de centralización de la autoridad estatal. Por tanto, el liberalismo fue aplicado en un contexto hostil que, en algunos casos, engendró una fuerte oposición por parte de ideologías conservadoras. La interactuación entre la teoría (nacida en Europa) y la realidad latinoamericana moduló los resultados previstos por el discurso liberal y trajo consigo efectos colaterales no deseados y no previstos por la teoría. En este sentido, los años que transcurren entre 1820 y 1870 son una era de conflicto ideológico y confusión política. Fueron años en los que las doctrinas clásicas del liberalismo experimentaron serias modificaciones dentro de este contexto (Hale 1986).

En el caso colombiano, podemos considerar que es uno de los ejemplos en los que esta tendencia se da con mayor intensidad. Así pues, ¿Cuáles fueron

\footnotetext{
${ }^{3}$ Tomado de Fischer (2004: 186).

${ }^{4}$ Tomado de Pécaut (2004:25).

${ }^{5}$ Tomado de Pécaut (2004: 25).
} 
las características que han presidido el peculiar recorrido histórico en la construcción del Estado-nación colombiano y de su territorialidad? ${ }^{6}$.

En primer lugar, no se ha elaborado un concepto fuerte de la Unidad Nacional. Entre los factores responsables de esta carencia podemos citar a la geografía física y orografía del país que configura un espacio complicado para las comunicaciones terrestres dificultando la cohesión. Asimismo, otro factor importante como ya se ha mencionado es la heterogeneidad de la composición étnica de la población con la presencia de minorías en buena parte del territorio nacional. En efecto, de acuerdo con Telban (1988) Colombia está dividida en cinco regiones naturales (Andina, Atlántica, Pacífica, Orinoquía y Amazonía) y encontramos presencia de minorías en 24 de los 32 departamentos del país, esto es, hay presencia indígena en el 75\% del territorio nacional. Estas minorías indígenas configuran un mosaico de 12 familias lingüísticas y 71 grupos étnicos. En estas condiciones, la generación de una identidad nacional robusta fue imposible de crear y más bien lo que sucedió fue la proliferación de identidades locales. Buen ejemplo de estas dificultades es lo que ocurrió con Panamá, paradigma que exhibe las falencias de la identidad nacional. Como es bien sabido, los Estados Unidos aprovecharon el sentimiento identitario de la población del territorio de Panamá para en 1903 promover la secesión de este territorio, hasta ese momento parte de Colombia, y su proclamación en República. Es indudable que sin la intervención norteamericana motivada por los intereses económicos derivados de la futura construcción del canal no es entendible la secesión de Panamá. Pero de igual manera, también no hay dudas de que, sin la existencia de un sentimiento de identidad local panameño en pugna con la identidad nacional colombiana, la secesión e independencia de Panamá no hubiera sido posible.

En segundo lugar, no se alcanzó a constituir una imagen fuerte de soberanía nacional ni a desarrollarla en la práctica. El comienzo de la "Gran Colombia" trajo consigo la desmembración del proyecto original bolivariano en tres repúblicas. A partir de este decepcionante comienzo, la trayectoria de Colombia como república a lo largo del siglo XIX fue la crónica de la lucha entre las elites bogotanas y las elites provinciales por ver quien imponía su hegemonía. Las guerras civiles colombianas pueden ser vistas como expresión del fracaso por imponer un consenso nacional unificador, así como también son expresión de la fortaleza de los intereses regionales y del caudillismo localista.

En tercer lugar, más que el Estado fueron los partidos políticos y la Iglesia Católica las instituciones creadoras del sentimiento de pertenencia nacional. Se trata, de nuevo, de una tendencia propia del liberalismo decimonónico latinoamericano. Tal como sostiene Hale (1986), uno de los elementos de

${ }^{6}$ Para la elaboración de esta caracterización recogemos los análisis de Pécaut (2004) y Fischer (2004).

Araucaria. Revista Iberoamericana de Filosofía, Política y Humanidades, año 20, no 39. Primer semestre de 2018. Pp. 341-363. ISSN 1575-6823 e-ISSN 2340-2199 doi: 10.12795/araucaria.2018.i39.17 
pugna entre el liberalismo y el conservadurismo fue la apuesta liberal por el laicismo del estado. El Estado laico se componía de individuos libres, iguales ante la ley. La lealtad de los ciudadanos debía ser dirigida y encauzada hacia el Estado y la Nación y no hacia la Iglesia u otras corporaciones, reminiscencia de la antigua sociedad colonial. Pero en Colombia la debilidad del Estado frente a instituciones como los partidos liberal y conservador y a la Iglesia Católica dio como resultado que el espacio de construcción de la identidad nacional fuera ocupado por estas instituciones.

Y el problema reside en que tanto los partidos políticos como la Iglesia Católica responden no al interés general sino a visiones particularistas y a identidades no inclusivas. Es decir, una identidad nacional construida sobre "lo liberal", "lo conservador" o "lo católico" da lugar a un juego de exclusiones que deja fuera de la nación a aquellos compatriotas que no son conservadores, liberales y/o católicos en función de cuál sea la matriz identitaria desde la que se construya y defina el "ser nacional". La construcción de identidades siempre es una operación de inclusión y exclusión pues construye un "nosotros" y un "ellos". Pero si esta operación es definida y protagonizada por el Estado entonces cabe la posibilidad de que el juego de inclusión abarque a todos los compatriotas independientemente de su cosmovisión ideológica o religiosa.

Es decir, un proyecto de construcción nacional llevado a cabo por un Estado liberal decimonónico coherente ${ }^{7}$ da como resultado que el cleavage de "lo nacional" abarque a toda aquella población que ha nacido dentro del territorio nacional independientemente de su filiación política o religiosa y por eso construye una dicotomía entre "los colombianos" y "los extranjeros". Mientras que un proceso de construcción de identidad nacional protagonizado por instituciones no estatales (partido político, iglesia) construye no una dicotomía sino una tipología en la que se distinguen varios grupos: "los verdaderos colombianos", "los colombianos traidores" y "los extranjeros". Esta distinción dentro de la población colombiana entre "verdaderos" y "traidores" indudablemente lleva a una particularización del sentimiento nacional que juega en su contra debilitándolo.

En cuarto lugar, el Estado colombiano es débil a raíz de la débil articulación de la nación colombiana. Las relaciones entre el Estado y la Nación son interdependientes y por lo tanto para que ambos términos del par conceptual estado-nación sean realidades consolidadas y eficaces, es necesario que los

\footnotetext{
7 Con la expresión "Estado liberal decimonónico coherente" me refiero a un Estado que ponga en práctica la teoría liberal clásica en la que éste se caracteriza por ser la institución que se ve a si misma como el garante del interés general y la Institución máxima que domina y ejerce la hegemonía en un territorio, no tolerando la competencia de otras instituciones y sometiéndolas jerárquicamente. Evidentemente se trata de un tipo ideal que, a la hora de tomar cuerpo en la realidad de la construcción de los países concretos, no solo en América Latina sino en occidente, dio lugar a distintas situaciones de mayor o menor autonomía estatal o, si se quiere, de mayor o menor sometimiento del Estado a otras instituciones como partidos políticos e iglesias.
} 
dos gocen, si se me permite la expresión, de buena salud. Y como venimos analizando, el proceso de conformación de la nación colombiana ha exhibido históricamente de falencias y debilidades. La heterogeneidad que es una característica constitutiva de la sociedad civil colombiana desde sus orígenes no ha sabido ser resuelta de modo creativo, de tal manera que se constituyera en una fortaleza de la nación. En su lugar, como venimos analizando, esta diversidad ha sido tradicionalmente percibida más como un problema al que había que poner remedio a través de la homogeneización. Y ese proceso de difuminación de la diversidad, en un contexto en el que las identidades se concebían en términos de dominio y exclusión sin lugar para el consenso y la recreación a través de la combinación de los distintos elementos identitarios, ha significado una debilidad congénita que ha conformado a la nación colombiana como desarticulada.

Por último $\mathrm{y}$, en quinto lugar, el Estado no ha conseguido establecer un espacio de dominio duradero con presencia de la Administración en todas las regiones del país. Y, además, donde la Administración está presente goza de bajos niveles de legitimidad debido a la corrupción y la arbitrariedad. La disputa por el "monopolio de la violencia organizada" por otros actores no estatales como insurgencias, paramilitares y narcotráfico expresa a partes iguales la debilidad del Estado y su falta de legitimidad a ojos de sectores significativos de la población colombiana. Es difícil de dilucidar si es la falta de presencia del Estado la que propicia que los actores no estatales ocupen el espacio que le correspondería a éste o, por el contrario, es la capacidad y el poder de estos actores estatales el que expulsa al Estado o no le deja ejercer sus funciones en amplias zonas del territorio nacional.

Probablemente sea una mezcla de ambos factores, pero el resultado es inequívoco: la ausencia del Estado. Como apunta Hidrum Zinecker ${ }^{8}$, el Estado colombiano es un "conjunto de espacios fragmentados marcados por estructuras paralelas entre poderes locales o segmentarios”. Así, Colombia es un país de regiones en el que se pone de manifiesto la fragmentación geográfica del país con una escasa colonización del territorio estatal y una concentración de la masa de población en pocos asentamientos. Además, la integración diferenciada de cada región en el comercio mundial desde 1850 hizo que las desigualdades se hicieran aún mayores. No se formó una clase dirigente nacional homogénea y la ausencia de objetivos comunes llevó a que prevalecieran los intereses políticos regionales (Fischer 2004: 191).

En síntesis, la siguiente descripción de Gabriel García Márquez (2002: 83, $209)^{9}$, referida a la primera mitad del siglo XX, dibuja gráficamente la realidad a la que me estoy refiriendo:

\footnotetext{
${ }^{8}$ Tomado de Fischer (2004: 159)
}

9 Tomado de Suárez (2004: 254) 


\begin{abstract}
"La provincia tenía la autonomía de un mundo propio y una unidad cultural compacta y antigua, en un cañón feraz entre la Sierra Nevada y la sierra de Perijá, en el Caribe colombiano. Su comunicación era más fácil con el mundo que con el resto del país, pues su vida cotidiana se identiñcaba mejor con las Antillas por el tranco fácil con Jamaica o Curaçao, y casi se confundía con la de Venezuela por una frontera de puertas abiertas que no hacía distinciones de rangos y colores. Del interior del país, que se cocinaba a fuego lento en su propia sopa, llegaba apenas el óxido del poder: las leyes, los impuestos, los soldados, las malas noticias incubadas a dos mil quinientos metros de altura y a ocho días de navegación por el río Magdalena [...] Por obra del centralismo, todo el que aspiraba a una beca tenía que ir a Bogotá, mil kilómetros en ocho días de viaje que costaba casi tanto como tres meses en el internado de un buen colegio".
\end{abstract}

En una realidad como ésta no es extraño que se levantaran voces que plantearan como solución al mosaico territorial colombiano la idea de la descentralización. Así, son precisamente estas diferencias regionales las que abren paso al proyecto descentralizador. El concepto de Estado Federalista fue introducido en una fecha tan temprana como 1863. A partir de este momento, la polémica Unitarismo vs. Federalismo se constituirá en una suerte de oscilación pendular que orientará el debate territorial cuyos hitos fundamentales serán la introducción del principio del Estado Central a partir de 1886 durante la hegemonía conservadora y la recuperación del principio de la descentralización en la Constituyente de 1991.

De acuerdo con Gómez (2011: 35) el modelo de organización territorial de la constitución de 1991 fue el resultado de un consenso articulado en torno a tres posiciones discursivas: federalistas, regionalistas y centralistas. Estas posiciones reflejaban los tres relatos que históricamente habían sostenido la pugna por alzarse con la hegemonía en la construcción territorial de Colombia. El federalismo no presentó una única voz siendo posible distinguir entre quienes privilegiaban en su análisis a la región como el ente territorial que podría definir cuáles serían sus relaciones con los Departamentos y aquellos otros que sostenían que los diversos entes territoriales (nación, departamentos y municipios) tenían que ser claramente definidos en cuanto a sus competencias, niveles de autogobierno y mecanismos de coordinación. Por su parte, el regionalismo se centraba en el reconocimiento de las particularidades y lealtades localistas mientras que el discurso centralista propugnaba el fortalecimiento de las capacidades del nivel nacional y una mayor presencia del Estado en todos los rincones del territorio. Finalmente, la fórmula que permitió satisfacer las pretensiones de las diversas posiciones y que concitó el acuerdo consistió en afirmar la unidad territorial del país haciéndola compatible con la regionalización y provincialización del país, manteniendo la existencia de los niveles locales de 
la administración local existente tales como los departamentos y los municipios y abriendo la puerta a la conformación de nuevos entes territoriales a través de la asociación de departamentos.

Hay acuerdo entre los analistas en considerar que la solución adoptada tiene más de Estado Unitario que de Estado Federal por cuanto establece una distinción clara entre lo político y lo administrativo. De este modo, el ámbito del poder político no se fragmenta, sino que corresponde en exclusiva al nivel central, esto es, a las autoridades nacionales. Donde se produce una descentralización es en la Administración Pública al establecerse una jerarquía de competencias administrativas que se corresponden con los niveles de la administración (nacional, departamental y municipal). Por tanto, existe una sola y única soberanía, un solo centro del poder político y una sola competencia legislativa pero múltiples centros de decisión administrativa (Gómez 2011: 3536).

La Constitución de 1991 establece en su artículo 1 que "Colombia es un Estado social de derecho organizado en forma de República unitaria, descentralizada, con autonomía de sus entidades territoriales". Por tanto, a través de los conceptos descentralización y autonomía se expresa el complejo consenso alcanzado entre las posiciones anteriormente citadas. Así se huye expresamente del término "federal" y se afirma la unidad del territorio nacional, para recoger los intereses del discurso centralista, mientras que simultáneamente se hace un guiño a las posiciones federalistas y regionalistas al hacer referencia a la descentralización y la autonomía.

Tres son los artículos que desarrollan el modelo de organización territorial: el 206, el 306 y el 307. El artículo 206 establece que "la ley podrá darles el carácter de entidades territoriales a las regiones y provincias. Dos o más departamentos podrán constituirse en regiones administrativas y de planificación". Por su parte los artículos 306 y 307 plantean el proceso a seguir para conformar las regiones o provincias: en primer lugar, se conforman como Regiones Administrativas de Planificación (RAP) con el objetivo de procurar el desarrollo socioeconómico de este territorio y posteriormente una RAP puede dar el paso a transformarse en Región de Entidad Territorial (RET), figura que supone una mayor autonomía frente al gobierno central. El proceso para configurar una RAP puede demorarse varios años y su paso a RET aún demoraría más tiempo. En definitiva, el procedimiento establecido en la constitución de 1991 es muy rígido y hace que la puesta en marcha del proceso de regionalización sea muy lenta. (Gómez 2011: 38). 


\section{La Ley de Ordenamiento Territorial de 2011}

En la Constitución de 1991 se remite a una futura Ley de Ordenamiento Territorial que desarrolle la regionalización a la que la carta magna hace referencia. Para darnos una idea de la dificultad que este proceso de organización territorial ha atravesado baste señalar que entre los años 1992 y 2010 se presentaron 19 proyectos de Ley Orgánica de Ordenamiento Territorial que fueron archivados. Es decir, en dieciocho años se presentaron diecinueve proyectos legislativos. Claramente esto es un indicador de la fragilidad del consenso constitucional alcanzado en Asamblea Nacional constituyente de 1991. La regionalización y descentralización es un tema altamente sensible para la cultura política colombiana y se hace muy difícil evolucionar desde las tradicionales posiciones centralistas hacia la autonomía de los distintos niveles del territorio. Finalmente, en junio de 2011, se logró aprobar la ley 1454 que es la normativa vigente en la actualidad y que establece las directrices por las que se está desarrollando la ordenación del territorio colombiano.

¿Cuáles son las características que podemos destacar del modelo territorial que en esta ley se contiene? (Duque 2012). En primer lugar, no desarrolla los objetivos enunciados en la constitución. Así, aunque la constitución del 91 autorizaba a transformar en entidades territoriales a Provincias, Regiones y Entidades Territoriales Indígenas además de posibilitar la autorización para la creación de nuevos Departamentos, de todos estos objetivos solo la creación de nuevos departamentos es contemplada por la Ley 1454 mientras que la transformación en entidades territoriales es remitida para su regulación a otra ley. En segundo lugar, no autoriza la creación de nuevas categorías de entidades territoriales. En tercer lugar, no establece con nitidez el deslinde de competencias entre el nivel central y los niveles intermedios y locales. El texto renuncia a establecer el mapa de competencias políticas y administrativas y se limita a establecer una distribución de funciones en materia de ordenamiento físico del territorio. En cuarto lugar, su propósito fundamental es la creación de una serie de figuras asociativas territoriales de naturaleza administrativa.

La norma es frágil y establece una pugna entre región y departamento. La consolidación de la región significa el debilitamiento de los departamentos. La ley deja intactos a los departamentos y lo que propone es un acuerdo voluntario, una alianza entre entidades territoriales para constituir regiones con lo que desvincula la dinámica socioespacial de los territorios de tal manera que la creación de regiones se basa en criterios puramente tecnocráticos que no tiene porqué corresponderse con dinámicas históricas ni socioespaciales. 


\section{La agenda de paz y la territorialización}

En el texto definitivo de los Acuerdos de Paz, tras la renegociación que se produce como consecuencia del triunfo del no en el referéndum celebrado el 2 de octubre de 2016, en el Preámbulo se establece que son tres los ejes fundamentales que lo inspiran: el enfoque territorial, diferencial y de género. Por lo que se refiere al enfoque territorial, éste se define así:

"El enfoque territorial del Acuerdo supone reconocer y tener en cuenta las
necesidades, características y particularidades económicas, culturales y sociales
de los territorios y las comunidades, garantizando la sostenibilidad socio-
ambiental; y procurar implementar las diferentes medidas de manera integral
y coordinada, con la participación activa de la ciudadanía. La implementación
se hará desde las regiones y territorios y con la participación de las autoridades
territoriales y los diferentes sectores de la sociedad." (Acuerdo de La Habana
2016: 6).

De la anterior definición se deduce que el concepto "Enfoque Territorial" consiste en el reconocimiento de que la realidad colombiana está configurada por un conglomerado plural y diverso de realidades socioespaciales. Y que, por lo tanto, la aplicación de las medidas que se contienen en las más de 300 páginas de los Acuerdos de Paz tienen que tener en cuenta esta situación de tal manera que su implementación no puede tener una lógica uniformizadora para todo el Estado sino que por el contrario tiene que adecuarse al perfil y la realidad que cada escenario demande. Es más, que hay que desechar la tradicional lógica centralista en la que el centro (Bogotá) impone los ritmos y directrices y la periferia (las regiones) acata sin capacidad de decisión. Por eso se establece que la puesta en marcha del Acuerdo de Paz tendrá que contar con la participación de las autoridades territoriales.

Hasta aquí no hay nada que objetar. Por el contrario, se trata de un reconocimiento por parte de los actores firmantes del Acuerdo (Gobierno e Insurgencia) de la necesidad de poner fin al secular olvido que han sufrido las regiones como consecuencia del histórico sesgo centralista que ha presidido las actuaciones del Estado Colombiano desde la Independencia. Sin embargo, si seguimos profundizando en el texto del Acuerdo de La Habana, encontramos que en su discurso el uso que se hace del campo semántico "territorio" es peculiar. En efecto, reproduzcamos algunas citas seleccionadas ${ }^{11}$ :

\footnotetext{
${ }^{10}$ Un campo semántico es un conjunto de palabras o elementos significantes con significados relacionados. Los campos semánticos son conjuntos de palabras, cuyo significado tienen algo en común, de ahí que se le llame campo, aunque cada palabra tenga su propio significado.

${ }^{11}$ Aunque pueda parecer que estas citas son demasiadas se ha procedido a una selección de las mismas puesto que el texto del Acuerdo se encuentra lleno de muchas otras que reiteran el uso del campo semántico "territorio" en el sentido particular que estamos poniendo de manifiesto.
} 
"La integración implica además la interrelación, el engranaje y la articulación de lo local, lo territorial, y lo nacional. En tal sentido el PNIS se integrará a los planes de desarrollo en los diferentes niveles del ordenamiento territorial" (Acuerdo de La Habana 2016: 103).

"La política frente al consumo de drogas ilícitas debe ser una prioridad y una política de Estado que requiere, entre otros, el fortalecimiento de las capacidades, tanto nacionales como territoriales, en el marco del sistema de protección social, y la disposición correspondiente de recursos" (Acuerdo de La Habana 2016: 116).

"Integración territorial e inclusión social: las medidas que se adopten en la implementación deben promover la integración de los territorios al interior de las regiones y la integración de éstas en el país, así como la inclusión de las diferentes poblaciones y comunidades, en particular de las más afectadas por el conflicto y las que han vivido en condiciones de pobreza y marginalidad (...).

Se reafirma el fundamento constitucional según el cual el Estado colombiano es descentralizado administrativamente y que los Entes Territoriales tienen autonomía, bajo los principios de concurrencia, coordinación y subsidiariedad, y por tanto se garantizará que la implementación se haga en coordinación y con el concurso de las autoridades locales. La implementación del Acuerdo se deberá realizar con pleno respeto de las competencias de las autoridades territoriales, sin detrimento de lo acordado.

En particular, las medidas deben promover el fortalecimiento de las capacidades de gestión de los departamentos, municipios y demás entidades territoriales, de manera que puedan ejercer el liderazgo en la coordinación de planes y programas necesarios en la construcción de la paz; y la articulación de las autoridades nacionales, departamentales y municipales para garantizar que actúen de manera integral, coordinada, articulada y ordenada en los territorios" (Acuerdo de La Habana 2016: 194).

Del anterior recuento de citas se obtiene que los términos que componen el campo semántico "territorio" está integrado por las siguientes palabras y expresiones: "territorios, comunidades, regiones, lo local, lo territorial, lo nacional, país, poblaciones, Entes Territoriales, autoridades territoriales, autoridades locales, departamentos, municipios, entidades territoriales, autoridades nacionales, autoridades departamentales, autoridades municipales".

Si además de hacer el mero recuento cuantitativo, nos ocupamos de examinar analíticamente cual es el uso que se hace de cada una de estas palabras, vemos que no hay una definición rigurosa para cada uno de estos significantes. Y el resultado de esta falta de definición es una ambigüedad en el uso de las palabras que en función del párrafo del texto en el que aparecen citadas pueden aludir a diferentes contenidos. Por ejemplo, en la primera de las citas se establece una 
división entre "lo local, lo territorial y lo nacional". Esta división contiene un error puesto que "lo local" es un nivel o división del territorio nacional por lo que no tiene sentido contraponer "lo local", "lo territorial" y "lo nacional" como distintos niveles en la ordenación del territorio.

Si continuamos con el análisis de la segunda cita, se observa el mismo error por cuanto se habla del "fortalecimiento de las capacidades, tanto nacionales como territoriales" volviendo a contraponer "lo nacional" con "lo territorial" cuando la realidad espacial nacional es también, por definición, una categoría territorial. Finalmente, en la tercera de las citas se puede ver como se habla de "promover la integración de los territorios al interior de las regiones y la integración de éstas en el país" y de nuevo se emplea incorrectamente la contraposición de "territorio" a "regiones" y "país" que igualmente son categorías que se definen territorialmente.

En definitiva, el campo semántico "territorio" está mal utilizado. No hay una definición correcta del ordenamiento territorial del Estado Colombiano. Un empleo adecuado del "Enfoque Territorial" en los Acuerdos de La Habana tendría que haber comenzado por plantear que de acuerdo con la Constitución de 1991 en su artículo 286 se establecen las siguientes divisiones espaciales: departamentos, regiones, provincias, distritos, municipios y territorios indígenas.

A continuación, y una vez definidos cuales son los distintos niveles, habría que describir de acuerdo a sus competencias cuales son los roles que le caben a cada nivel administrativo en la implementación de cada punto de la agenda de paz. En lugar de esta forma de proceder, da la impresión de que los significantes "territorio" y "territorial" se utilizan para referirse a todos aquellos niveles administrativos más pequeños que la capital del país o que las capitales departamentales. Es decir, el par conceptual "rural/urbano" aparece sustituido en el texto del Acuerdo de La Habana por el binomio "territorial/nacional". De tal manera que "territorial=rural" y "nacional=urbano". Lo cual es una manera de definir la periferia (el campo) desde el centro (la ciudad) y así cometer errores al unificar todo lo que no pertenece al mundo urbano bajo la misma etiqueta de "los territorios" sin darse cuenta de que hay toda una constelación de territorios y una jerarquía entre las diversas realidades socioespaciales que se invisibilizan cuando se define por lo que no es (lo urbano) y no por lo que es.

La fuerza ilocucionaria ${ }^{12}$ que late detrás del texto del Acuerdo de La Habana es muy clara: pone de manifiesto la necesidad de que los intereses y demandas del mundo rural, históricamente ignorados y marginados, sean tenidos en cuenta y que la aplicación de los Acuerdos venga a poner fin, en

\footnotetext{
12 Para Austin, un acto lingüístico ilocucionario es aquel en el que diciendo se pretende hacer algo. Para este autor, no es posible una verdadera captación de la totalidad del significado de los discursos si no se tiene en cuenta la fuerza ilocucionaria de los mismos: las verdaderas intenciones que tenía el autor cuando escribió ese texto. Por eso una pregunta fundamental que hay que responder en cualquier investigación es ¿Qué quiso hacer el autor al escribir el texto? (Harto de Vera 2005: 222-223)
} 
alguna medida, al secular olvido al que Bogotá ha sometido al agro colombiano y sus habitantes.

No podía ser de otra manera puesto que las FARC a diferencia de otras guerrillas como el M19, de naturaleza urbana, o el grupo insurgente "Quintín Lame", de naturaleza indígena, es una insurgencia de carácter campesino. El problema estriba en que no basta con identificar el origen del conflicto en la situación del campo para poder lograr escapar de las lógicas hegemónicas que históricamente han sido y son urbanas. Una lógica urbana que es por definición centralista y que ha conformado a lo largo de los más de dos siglos de vida republicana tras la Independencia una manera de percibir la realidad en la que el centro (la ciudad) es quien genera la epistemología que define como explicar la realidad (el campo y la ciudad).

Es decir, los dos actores (gobierno e insurgencia), más allá de las declaraciones retóricas, en realidad están presos de la lógica centralista. Esta lógica hace que ambos actores no tengan un proyecto nacional. En efecto, el gobierno piensa para y desde la ciudad y no tiene en cuenta los intereses y la realidad de las regiones. Y viceversa, la insurgencia debido a la impronta cultural de lo campesino (guerrilla campesina) piensa para y desde las regiones pero ignora a la ciudad. El resultado es que ambos actores reproducen la lógica excluyente campo/ciudad porque no son capaces de escapar de este modo tradicional de pensar. Ambos expresan la realidad de uno de los dos extremos del binomio, pero son incapaces de superar la lógica binomial campo/ciudad como hubiera requerido un enfoque "de estado". Es decir, por enfoque "de Estado" hay que entender una forma de pensar la nación desde la complejidad que significan los distintos niveles territoriales que la integran teniendo en cuenta las interrelaciones tanto cooperativas como competitivas que se dan entre los distintos niveles socioespaciales que integran la totalidad del territorio nacional.

La Agenda de paz de los Acuerdos de La Habana contiene 6 puntos que son los capítulos en los que se desgrana el contenido de los Acuerdos de Paz: 1) Desarrollo Agrario Integral 2) Participación Política 3) Fin del conflicto 4) Drogas ilícitas 5) Víctimas y 6) Implementación, verificación y refrendación ${ }^{13}$. En un análisis cuantitativo se obtiene que la extensión del Acuerdo de La Habana se despliega de acuerdo a lo que se contiene en el cuadro I.

${ }^{13}$ A estos seis puntos se le añade un capítulo de Protocolos y Anexos. El Acuerdo de Paz sin el capítulo de Protocolos y Anexos tiene 218 páginas que son las que se han tomado en cuenta para realizar los cálculos que se muestran en el Cuadro I. 


\section{Cuadro I: El Acuerdo de La Habana en cifras}

\begin{tabular}{|l|c|c|c|}
\hline CAPÍTULO & $\begin{array}{l}\text { PAGINAS } \\
\text { INICIAL } \\
\text { Y FINAL }\end{array}$ & $\begin{array}{l}\text { NÚMERO DE } \\
\text { PÁGINAS }\end{array}$ & $\begin{array}{l}\text { PORCENTAJE } \\
\text { RESPECTO } \\
\text { AL TOTAL }\end{array}$ \\
\hline Preámbulo & $1-5$ & 4 & $1,83 \%$ \\
\hline Introducción & $6-9$ & 3 & $1,37 \%$ \\
\hline 1) Reforma Rural Integral & $10-34$ & 24 & $11 \%$ \\
\hline 2) Participación Política & $35-56$ & 21 & $9,63 \%$ \\
\hline 3) Fin del Conflicto & $57-97$ & 40 & $18,34 \%$ \\
\hline 4) Drogas Ilícitas & $98-123$ & 25 & $11,46 \%$ \\
\hline 5)Víctimas & $124-192$ & 68 & $31.19 \%$ \\
\hline $\begin{array}{l}\text { 6) Implementación, } \\
\text { verificación y refrendación }\end{array}$ & $193-218$ & 25 & $11.46 \%$ \\
\hline
\end{tabular}

Fuente: Elaboración propia.

Como puede observarse de los datos contenidos en el Cuadro I, el núcleo fundamental del Acuerdo se contiene en los capítulos 5 y 3 dedicados a "Víctimas" y "Fin del conflicto", respectivamente, puesto que entre ambos suman casi la mitad de las páginas del texto (49.53\%). El capítulo 5, el más extenso de todos, está estructurado en tres puntos que son, a) "Sistema Integral de Verdad, Justicia, Reparación y No Repetición” b) “Jurisdicción Especial para la paz" y c) "Compromiso sobre Derechos Humanos". Por su parte, el capítulo 3, el segundo más extenso, recoge dos puntos: a) Cese al fuego y hostilidades y b) Reincorporación de las FARC-EP a la vida civil.

Respecto al concepto de "Enfoque Territorial", una vez que el contenido del mismo es definido en el Preámbulo y en la Introducción, los dos capítulos en los que aparece más citado son el capítulo 1 dedicado a la "Reforma Rural Integral" y el capítulo 5 dedicado a "Víctimas"14. Por lo que se refiere al capítulo 1 , se establecen toda una serie de medidas destinadas a dotar al campesinado colombiano de tierra e insumos para poner fin a la situación secular de miseria y abandono. El instrumento diseñado para poner en marcha la Reforma Rural Integral son los denominados "Planes de Desarrollo con Enfoque Territorial (PDET)".

14 En el capítulo 1, el enfoque territorial aparece en 10 páginas mientras que en el capítulo 5, se le dedican 9 páginas. En el resto de los capítulos del acuerdo también aparece citado el enfoque territorial, aunque con un menor énfasis lo que se traduce en que se le dedican un número menor de páginas de acuerdo al siguiente cómputo:

Capítulo 2: 3 páginas.

Capítulo 3: 5 páginas.

Capítulo 4: 6 páginas.

Capítulo 6: 8 páginas.

Araucaria. Revista Iberoamericana de Filosofía, Política y Humanidades, año 20, no 39. Primer semestre de 2018. Pp. 341-363. ISSN 1575-6823 e-ISSN 2340-2199 doi: 10.12795/araucaria.2018.i39.17 
El objetivo de los PDET consisten en "la erradicación de la pobreza y la satisfacción plena de las necesidades de la ciudadanía de las zonas rurales, de manera que se logre en el menor plazo posible que los campesinos, las campesinas y las comunidades, incluidas las afrodescendientes e indígenas, ejerzan plenamente sus derechos y se alcance la convergencia entre la calidad de vida urbana y la calidad de vida rural, respetando el enfoque territorial, el enfoque de género y la diversidad étnica y cultural de las comunidades" (Acuerdo de La Habana 2016: 12).

En definitiva, los PDET consisten en la elaboración de un repertorio de políticas públicas destinadas a dinamizar aquellos lugares del agro colombiano que exhiben una situación de mayor pobreza y marginalidad, así como aquellas zonas particularmente afectadas por las consecuencias y dinámicas de la guerra y de la presencia de cultivos ilícitos. La participación de la población de estos territorios es citada explícitamente como un pilar fundamental para asegurar tanto la eficacia en el diagnóstico y en las soluciones como para respetar la idiosincrasia y particularidades identitarias. Finalmente se establece que "el gobierno nacional destinará los recursos necesarios para garantizar el diseño y ejecución de los planes de acción para la transformación estructural, con el concurso de las entidades territoriales" (Acuerdo de La Habana 2016: 23).

En cuanto al capítulo 5, el Enfoque Territorial está presente en cada uno de los tres puntos, señalados más arriba, en los que este capítulo se estructura. Sin embargo, y a diferencia de lo que ocurre en el capítulo 1, las referencias a la importancia del territorio en la implementación del contenido de este capítulo tienen un carácter más vago y menos concreto. Se limitan a subrayar la importancia de que las políticas públicas destinadas a reparar a las víctimas del conflicto tengan en cuenta las dinámicas territoriales lo cual es tanto como decir que el conflicto no afectó a todos los territorios por igual y que por lo tanto una parte importante de la condición de cada víctima es tener en cuenta cual fue el escenario en el que su afectación como víctima se produjo.

Una vez analizado cual es el lugar que al Enfoque Territorial se le asigna dentro del texto de los Acuerdos de La Habana podemos afirmar que el contenido con el que este concepto se emplea está definido por un marcado carácter de puesta en valor del mundo rural frente al urbano. La imagen que trasluce este Enfoque Territorial es la de que el conflicto se inició en el campo y es en el campo donde debe solucionarse. Que la mayor parte de las víctimas se concentran en las áreas rurales y que, por lo tanto, es en la periferia rural en donde los recursos deben concentrarse.

De nuevo, volvemos a encontrar que el significante "territorial" se utiliza como sinónimo de "ruralidad". Y, a nuestro juicio, esto conlleva restringir el significado de "territorial" a solo una parte del territorio nacional puesto que dentro de este territorio se encuentran tanto las zonas rurales como las urbanas, 
tanto el centro como la periferia. Un "Enfoque Territorial" correctamente utilizado debería haber contemplado una perspectiva global, si se quiere "nacional", que contemplara la totalidad del espacio (lo rural y lo urbano). Y lo que es más importante, las relaciones que ligan y conforman la interactuación entre centro(s) y periferia(s). Un enfoque así planteado permitiría establecer una actuación en términos de políticas públicas que abordara el conjunto de la problemática de los distintos niveles territoriales que conforman la realidad socioespacial de Colombia. Por el contrario, la definición parcial contenida en los Acuerdos de La Habana, más allá de sus buenas intenciones, tiende a perpetuar las relaciones tradicionales entre lo rural y lo urbano, entre centro(s) y periferia(s). Y ello porque no se contempla una dimensión fundamental para equilibrar y democratizar la interactuación entre los distintos territorios: la descentralización.

En efecto, si se realiza un análisis de contenido del campo semántico "descentralización" en el texto de los Acuerdos de La Habana obtenemos el siguiente resultado:

"La garantía de la provisión del servicio de asistencia integral, técnica y tecnológica (avances en los aspectos técnico-productivos, organizativos y sociales, de gestión, administración, informática, finanzas, mercadeo y capacitación) a la producción de la economía campesina, familiar y comunitaria, de manera descentralizada" (Acuerdo de La Habana 2016: 29).

"El diseño e implementación progresiva de un mecanismo de compras públicas para atender la demanda de las entidades y programas institucionales que, de manera descentralizada, fomente la producción local para apoyar la comercialización y absorción de la producción de la economía campesina, familiar y comunitaria" (Acuerdo de La Habana 2016: 31).

A estas dos citas se suma la última cita que se reprodujo más arriba a la hora de analizar el campo semántico "territorio" (Acuerdos de La Habana 2016: 194). Resulta particularmente significativo que en un texto que comprende más de doscientas páginas (sin contar los anexos) el campo semántico "descentralización” solo aparezca en tres páginas. Y cuando aparece, únicamente es para plantear la necesidad de que la aplicación de determinadas políticas públicas se haga teniendo en cuenta la escala territorial en la que se implementan.

Se trata de una referencia a todas luces insuficiente. Un tratamiento correcto de la cuestión de la descentralización hubiera requerido de una mayor profundidad y complejidad. Se debería haber definido con detalle cuales son los mecanismos presupuestarios con los que se va a dotar a cada nivel de la Administración para la puesta en marcha de las medidas contempladas en los Acuerdos de La Habana. En vez de esto se zanja la cuestión haciendo una 
alusión a que el Estado Colombiano es descentralizado porque así lo establece la Constitución de 1991. Sin embargo, la realidad de facto es que Colombia más allá de lo que proclame el ordenamiento jurídico vigente se caracteriza por ser uno de los Estados más centralistas en toda América Latina. En efecto, de acuerdo con Asela (2015: 71):

"el proceso de descentralización en Colombia en los últimos 20 años, trajo consigo grandes contribuciones para el país en el plano político, avances en participación democrática con la elección de alcaldes y gobernadores, prestación de servicios públicos de mayor calidad y con amplia cobertura, veedurías ciudadanas como mecanismo de vigilancia sobre las acciones correspondientes a la gestión pública, son solo algunos de los aspectos positivos del proceso en Colombia. A su vez en términos de autonomía fiscal y administrativa, es otro el panorama: retroceso en la asignación de recursos, pérdida de autonomía presupuestaria (Ley 715, 1176, Ley de Regalías) son algunas muestras del poder que aún ejerce el gobierno central sobre los entes territoriales y sus políticas".

El propio Departamento Nacional de Planeación (DNP), organismo gubernamental, elabora un índice denominado "Indicador de dependencia intergubernamental" que mide el grado de dependencia de los municipios y departamentos frente a los recursos transferidos por parte del gobierno central. Pues bien, en el caso de los municipios se tiene que para el año 2012, de un total de 1101 municipios, 913 tienen una dependencia mayoritaria de los recursos provenientes del gobierno central, 84 tienen una dependencia moderada y 104 son autosuficientes. Es decir, el 82, 92\% de los municipios son incapaces de mantener su actividad sin los recursos provenientes del gobierno central. Para el caso de los departamentos, en el periodo 2008-2012, de un total de 32 departamentos, 21 tienen una dependencia mayoritaria, 5 tienen una dependencia moderada y 6 departamentos son autosuficientes (Asela 2015: 72).

La conclusión de estos datos no da lugar a dudas: desde la entrada en vigor de la constitución de 1991 la descentralización en Colombia ha producido avances en términos políticos, pero en cuanto a la financiación y a los recursos la situación es claramente centralista.

\section{Conclusión: ¿Territorialización o Periferialización?}

La territorialización tal y como es entendida en Los Acuerdos de La Habana no es sino un sinónimo para referirse al mundo rural. Y la territorialización así entendida, sin que haya autonomía financiera, no es más que periferialización. Digámoslo claramente: la territorialización sin descentralización da como resultado la periferialización. Una periferialización en el sentido de marginalización por cuanto es la expulsión de la Agenda de Paz a los márgenes 
del sistema. Es hacer que la paz deje de ser un asunto de interés nacional para que sea una cuestión de interés periférico y marginal. En definitiva, es reforzar el imaginario del sentido común bogotano de que la guerra es cosa del campo: empezó allí y es allí donde debe terminar.

El riesgo que se corre con la periferialización de la paz es muy serio. Si el gobierno central cae en la tentación de utilizar el presupuesto como un arma política, podríamos vernos en la situación de que aquellos municipios rurales en los que la Fuerza Alternativa Revolucionaria del Común lograra posiciones de poder fueran asfixiados económicamente. Un escenario así llevaría indudablemente a una frustración de las expectativas de la población que ha apoyado con su voto un proyecto de cambio. Y el potencial fracaso de esta gestión no solo sería un fracaso de los alcaldes y alcaldesas del cambio, sino que claramente sería un fracaso de Colombia como nación capaz de construirse con una paz inclusiva.

A partir de esta frustración sería plausible pensar que se reforzarían las tendencias antipaz y proviolencia pudiendo darse un escenario de vuelta a la violencia por parte de algunos sectores. Pero a diferencia del pasado, en este caso no se trataría de violencia política sino de violencia delincuencial o común. No se contaría con la disciplina que una estructura política jerárquicamente organizada impone. Por el contrario, nos hallaríamos ante una violencia anómica de muy difícil resolución.

En términos comparados, el proceso de paz colombiano tiene dos experiencias en las que mirarse: El Salvador y Guatemala. Ambas constituyen la cara (El Salvador) y la cruz (Guatemala) de la implementación de un proceso de paz tras décadas de guerra. El episodio del referéndum de octubre de 2016 acercó dramáticamente la experiencia colombiana a lo sucedido hace diecisiete años en Guatemala. A partir de esa fecha esta tendencia a la "guatemalización" se ha visto reforzada con las serias dificultades por las que ha venido atravesando la aplicación de los Acuerdos de La Habana: ralentización legislativa de las reformas, asesinatos de líderes sociales, etc. Si se continúa con esta dinámica la periferialización de la paz sería ya el golpe de gracia a la mayor ventana de oportunidad política que se ha abierto para Colombia en décadas. 


\section{Referencias bibliográficas:}

AcuerdodeLaHabana(2016)disponibleenhttp://www.altocomisionadoparalapaz. gov.co/procesos-yconversaciones/Documentos\%20compartidos/24112016NuevoAcuerdoFinal.pdf, consultado el 20 de diciembre de 2017.

Asela, J. (2015). "La descentralización en Colombia, ¿realidad o espejismo? Consideraciones sobre la actualidad del proceso". Revista CEA, vol. 1, núm. 1, pp. 65-74.

Bergmann G. ([1953]2003). Logical Positivism, Language and the reconstruction of Metaphysics. Tegtmeier, Frnakfurt/Lancaster: OntosVerlag.

Bolívar S. (2001). "Mensaje del Libertador a la Convención de Ocaña del 1 de mayo de 1828". En Bolívar S. Proclamas y Discursos. Bogotá: FICA.

Boyer A. (2007). "Hacia una crítica de la razón geográfica". Universitas Philosophica, vol. 49, núm. 24, pp. 159-174.

Bushnell, D. (1996). Colombia. Una Nación a pesar de sí misma. Bogotá: Planeta.

Colom F. y Rivero A. (2015). El espacio político. Aproximaciones al giro espacial desde la teoría política. Barcelona: Anthropos.

Duque N. (2012). "Particularidades de la Ley Orgánica de Ordenamiento Territorial". Análisis Político, vol. 25, núm. 76, pp. 175-190.

Fischer T. (2004). "Estado débil sin territorialidad entera. Una visión histórica". VVAA. Dimensiones territoriales de la guerra y la paz. Bogotá: Universidad Nacional de Colombia.

Foucault, M. (1984) “Des espaces autres”. Architecture, Mouvement, Continuité, núm. 5, pp. 46-49.

García Márquez G. (2002). Vivir para contarla. Bogotá: Editorial Diana.

Hale C. (1986). "Political and social Ideas in Latin America: 1870-1930". En Bethell L (comp.) The Cambridge History of Latin America. Vol IV. Cambridge: Cambridge University Press.

Gómez Bello E. A. (2011). El ordenamiento territorial como alternativa de paz en Colombia, Facultad de Ciencias Políticas y Relaciones Internacionales, Pontificia Universidad Javeriana.

Disponible en https://repository.javeriana.edu.co/handle/10554/1415. Consultado el 24 de enero de 2018.

González F. (2006). Partidos, guerras e Iglesia en la construcción del Estadonación en Colombia (1830-1900). Medellín: La Carreta.

Harto de Vera F. (2005). Ciencia Política y Teoría Política contemporáneas: una relación problemática. Madrid: Trotta. 
Pécaut D. (2004). "Hacia la desterritorialización de la guerra y de la resistencia a la guerra". VV.AA. Dimensiones territoriales de la guerra y la paz. Bogotá: Universidad Nacional de Colombia.

Quesada F. (2016). "El giro espacial. Conquista y fetiche". Revista Europea de Investigación en Arquitectura, núm. 5, pp. 171-187.

Rorty R. (1990). El giro lingüístico. Barcelona: Paidós.

Soja E. (2008). Postmetrópolis. Estudios críticos sobre las ciudades y las regiones. Madrid: Traficantes de Sueños.

Soja E. (2009). "Taking space personally". En Warf B. and Arias S. (eds). The Spatial Turn. Interdisciplinary perspectives. Nueva York: Routledge.

Suárez, N. (2004) "Descentralización, conflictos y desigualdades socioespaciales". VV.AA. Dimensiones territoriales de la guerra y la paz. Bogotá: Universidad Nacional de Colombia.

Telbán B. (1988). Grupos étnicos de Colombia. Quito: ABYA-YALA.

Velázquez Ramírez A. (2013). "Espacio de lucha política: teoría política y el giro espacial". Argumentos, núm. 73, pp. 175-195

Warf B. and Arias S. (2009). The Spatial Turn. Interdisciplinary perspectives. Nueva York: Routledge. 
